\title{
THE ROLE OF NATIONAL CULTURAL VALUES WITHIN THE THEORY OF PLANNED BEHAVIOUR
}

Louise M. Hassan, Lancaster University, U.K. Edward Shiu, Bangor University, U.K.

\begin{abstract}
The theory of planned behaviour (TPB;Ajzen 1991) has received substantial research interest with a large number of reviews and meta-analyses concluding favourably on the ability of the TPB to explain intention and behaviour across a wide spectrum of contexts (e.g. Albarracin et al. 2001; Armitage and Conner 2001; Conner and Armitage 1998; Godin and Kok 1996; Sheppard et al. 1988). The TPB is an expectancy value model which states that human behaviour is a consequence of one's behavioural intention, which is in turn explained by one's attitude, subjective norm and perceived behavioural control regarding the behaviour.

One important area of interest which has not been adequately researched concerns the impact of national culture on the TPB constructs and the TPB relationships. Culture has been defined as 'the collective programming of the mind which distinguishes the members of one human group from another' (Hofstede 1980, p.25). Beyond individual differences, human dispositions and behaviours are influenced by the norms, beliefs and values of their cultural environment (Triandis 1989). Furthermore, cultural values shape not only one's behaviour but also one's perceptions of the self and of the social environment (Triandis 1989). To date, no systematic assessment of the impact of culture on the TPB has been undertaken. The present research examined the TPB in the context of smoking cessation with samples across 23 European Union Member States based on multi-level data analysis (hierarchical linear modelling) method.
\end{abstract}

At the individual level of analysis, our results showed that attitude $(B=-0.13, t=-5.11, p<.001)$, subjective norm $(B=0.05$, $t=3.64, p<.01)$ and self-efficacy $(B=0.33, t=10.83, p<.001)$ each had a significant $(p<.01)$ impact on intention. Examination of the variance components showed that there was significant variation across the 23 EU countries for the attitude - intention slope (variance component $=0.010, \chi^{2}(22)=66.07, p<.001$ ) and the self-efficacy - intention slope (variance component $\left.=0.016, \chi^{2}(22)=115.86, p<.001\right)$. However there was no evidence of a slope effect for subjective norm (variance component $=0.001, \chi^{2}(22)=32.40, p=.070$ ). Finally, examination of the variation in intercept, i.e. mean intention level across the $23 \mathrm{EU}$ countries, showed that significant variation existed (variance component $=.090, \chi^{2}(22)=$ $184.85, p<.001)$. Hofstede's cultural framework was used to assess between-country variation via cross level analysis. Hofstede's dimension of power distance explained $25 \%$ of variance in the attitude - intention slope while $53 \%$ of variance in the self-efficacy - intention slope was accounted for by both power distance and uncertainty avoidance.

Despite numerous meta-analyses on the TPB reported in the literature, few have considered the potential moderating effect of culture. For example, Albarracin et al. (2001) in their meta-analysis of the TRA and the TPB noted the country of each study but did not use this information to explain possible differences across the studies. Schepers and Wetzels (2007) considered culture as a moderator variable in their meta-analysis of the technology acceptance model and suggested but did not test the role of the Hofstede dimensions on the relationship between subjective norm and intention. As far as we are aware, our study marks a milestone in systematically assessing the impact of national culture on the TPB relationships. This research is thus important in addressing the central question as to whether the TPB can be considered to apply universally to humans (i.e. etic). The universal acceptance and application of the TPB has remained largely unchallenged, yet anecdotal evidence are accumulating to suggest that the TPB might be culture bound (i.e. emic). Our study has evidenced significant cross-national variations in the mean values of the TPB constructs and in the set of TPB relationships.

References Available Upon Request 